\title{
Stage specific transcriptome profiles at cardiac lineage commitment during cardiomyocyte differentiation from mouse and human pluripotent stem cells
}

\author{
Sung Woo Cho ${ }^{1,2, \#, *}$, Hyoung Kyu Kim ${ }^{2,3, \#}$, Ji Hee Sung ${ }^{2,3}$ E Jin Han ${ }^{2,3, *}$ \\ ${ }^{1}$ Division of Cardiology, Department of Internal Medicine, Inje University College of Medicine, Ilsan Paik Hospital Vision 21 Cardiac \& \\ Vascular Center, Goyang 10380, ${ }^{2}$ Cardiovascular and Metabolic Disease Center, Smart Marine Therapeutics Center, Inje University College \\ of Medicine, Busan 47392, ${ }^{3}$ Department of Physiology, Department of Health Sciences and Technology, BK21 Plus Project Team, Inje \\ University College of Medicine, Busan 47392, Korea
}

\begin{abstract}
Cardiomyocyte differentiation occurs through complex and finely regulated processes including cardiac lineage commitment and maturation from pluripotent stem cells (PSCs). To gain some insight into the genome-wide characteristics of cardiac lineage commitment, we performed transcriptome analysis on both mouse embryonic stem cells (mESCs) and human induced PSCs (hiPSCs) at specific stages of cardiomyocyte differentiation. Specifically, the gene expression profiles and the proteinprotein interaction networks of the $\mathrm{mESC}$-derived plateletderived growth factor receptor-alpha (PDGFR $\alpha)^{+}$cardiac lineagecommitted cells (CLCs) and hiPSC-derived kinase insert domain receptor (KDR) ${ }^{+}$and PDGFR $\alpha^{+}$cardiac progenitor cells (CPCs) at cardiac lineage commitment were compared with those of mesodermal cells and differentiated cardiomyocytes. Gene Ontology and Kyoto Encyclopedia of Genes and Genomes pathway analyses revealed that the genes significantly upregulated at cardiac lineage commitment were associated with responses to organic substances and external stimuli, extracellular and myocardial contractile components, receptor binding, gated channel activity, PI3K-AKT signaling, and cardiac hypertrophy and dilation pathways. Protein-protein interaction network analysis revealed that the expression levels of genes that regulate cardiac maturation, heart contraction, and calcium handling showed a consistent increase during cardiac differentiation; however, the expression levels of genes that regulate cell differentiation and multicellular organism development
\end{abstract}

*Corresponding authors. Sung Woo Cho, Tel: +82-31-910-7830; Fax: +82-31-910-7829; E-mail: drswcho@hanmail.net; Jin Han, Tel: +8251-890-6727; Fax: +82-51-894-5714; E-mail: phyhanj@inje.ac.kr ${ }^{\text {\#}}$ These authors contributed equally to this work.

https://doi.org/10.5483/BMBRep.2021.54.9.046

Received 1 April 2021, Revised 16 April 2021, Accepted 1 May 2021

Keywords: Cardiac lineage commitment, Cardiomyocyte differentiation, Pluripotent stem cell, Transcriptome profile decreased at the cardiac maturation stage following lineage commitment. Additionally, we identified for the first time the protein-protein interaction network connecting cardiac development, the immune system, and metabolism during cardiac lineage commitment in both mESC-derived PDGFR $\alpha^{+} \mathrm{CLCs}$ and hiPSC-derived $\mathrm{KDR}^{+} \mathrm{PDGFR} \alpha^{+}$CPCs. These findings shed light on the regulation of cardiac lineage commitment and the pathogenesis of cardiometabolic diseases. [BMB Reports 2021; 54(9): 464-469]

\section{INTRODUCTION}

Cardiomyocyte differentiation from pluripotent stem cells (PSCs) involves complex processes that are tightly regulated (1). For example, it was found that mesodermal cells that give rise to cardiomyocytes could also give rise to cells of endothelial, hematopoietic, and mural lineage (2). Cardiomyocyte differentiation from mesodermal cells generally involves cardiac lineage commitment (or fate determination) and maturation, which is a continuous process and of which the timing is difficult to define (3). Although cardiac lineage commitment, cell proliferation, and maturation occur simultaneously during the early period of cardiomyocyte differentiation, cell proliferation is the dominant action during this period. On the other hand, the proliferative capacity of the cells decreases and the maturation process becomes dominant during the late stage of differentiation $(3,4)$

It has been reported that cells expressing both the kinase insert domain receptor (KDR) and platelet-derived growth factor receptor-alpha (PDGFR $\alpha$ ) are populations of cardiac progenitor cells (CPCs) with cardiomyogenic potential $(5,6)$. Our group also previously described a novel population of PSC-derived PDGFR $\alpha^{+}$cardiac lineage-committed cells (CLCs), which while being actively proliferating cells, were also morphologically and functionally immature compared to differentiated cardiomyocytes $(7,8)$. However, the detailed biological processes and molecular mechanisms involved in 
cardiac lineage commitment have still not yet been fully determined (9). Therefore, to elucidate the genome-wide characteristics of cardiac lineage commitment, in this study, we compared the transcriptome profiles and the protein-protein interaction networks of highly upregulated genes in mouse embryonic stem cell (mESC)-derived PDGFR $\alpha^{+} \mathrm{CLCs}$ and human induced PSC (hiPSC)-derived $\mathrm{KDR}^{+} \mathrm{PDGFR} \alpha^{+} \mathrm{CPCs}$ with those of mesodermal cells and differentiated cardiomyocytes.

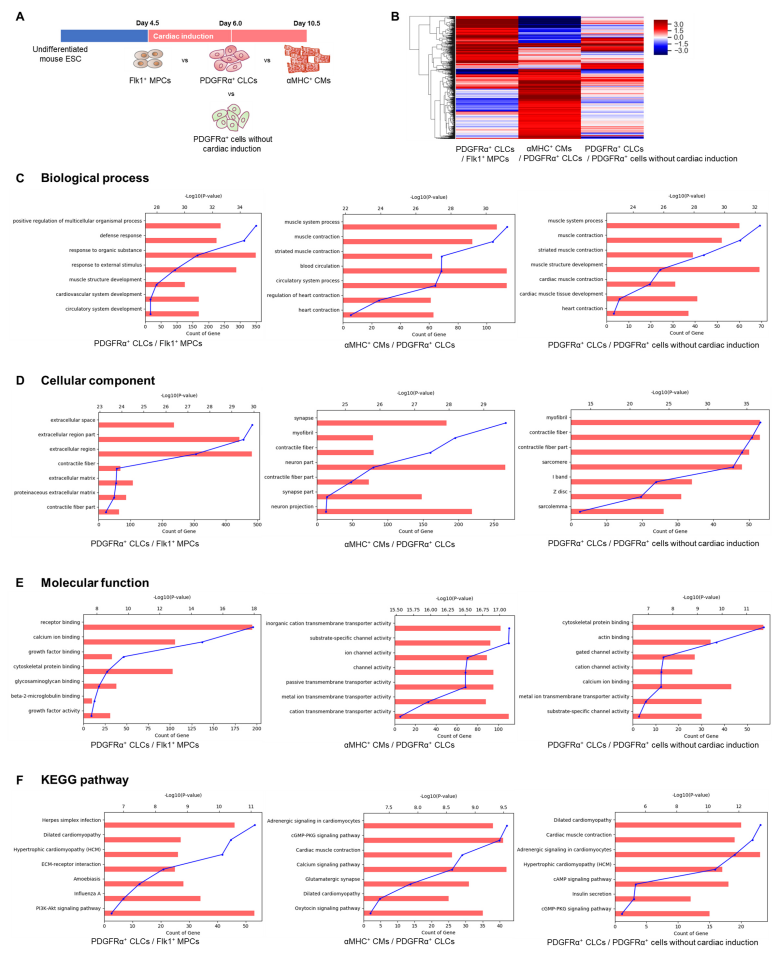

Fig. 1. Microarray transcriptome analysis of stage-specific cells during mESC-derived cardiac differentiation. (A) Sampling time points of mESC-drived cells: Flk1 ${ }^{+}$MPCs at day 4.5, PDGFR $\alpha^{+}$ $\mathrm{CLCs}$ and PDGFR $\alpha^{+}$cells without cardiac induction at day 6.0 , and $\alpha \mathrm{MHC}^{+}$cardiomyocytes at day 10.5. mESC: murine embryonic stem cell; Flk1 ${ }^{+}$MPCs: vascular endothelial growth factor receptor 2-expressing mesodermal precursor cells; PDGFR $\alpha^{+} \mathrm{CLCs}$ : plateletderived growth factor receptor-alpha-expressing cardiac lineagecommitted cells; $\alpha \mathrm{MHC}$ : alpha-myosin heavy chain. (B) Heatmap of the hierarchical clustering comparison of each developmental stage of cells derived from mESCs. (C-E) Gene Ontology analysis of the significantly upregulated genes at each developmental stage in cells derived from mESCs, showing the top $7 \mathrm{GO}$ terms in the (C) Bological Process, (D) Cellular Component, and (F) Molecular Function categories. (F) Kyoto Encyclopedia of Genes and Genomes pathway analysis of the significantly upregulated genes in each developmental stage of cells derived from mESCs; the top 7 pathways are shown.

\section{RESULTS}

\section{Clustering heatmap, Gene Ontology, and KEGG pathway analyses}

The differentially expressed gene (DEGs) patterns of the various transcriptomes were depicted using hierarchical clustering analysis in order to distinguish each developmental stage of the cells derived from the mESCs (Fig. 1B) and hiPSCs (Fig. 2B). Significantly upregulated genes in the mESC-derived cells were defined as those having a fold change $\geq 3.5$, normalized data $(\log 2)$ of 4 , and a P-value $<0.05$ and significant upregulation in the hiPSC-derived cells was defined as a fold change $\geq 2.0$, normalized data ( $\log 2)$ of 4 , and a p-value of $<0.05$. To determine the stage-specific role of the upregulated genes, Gene Ontology (GO) and Kyoto Encyclopedia

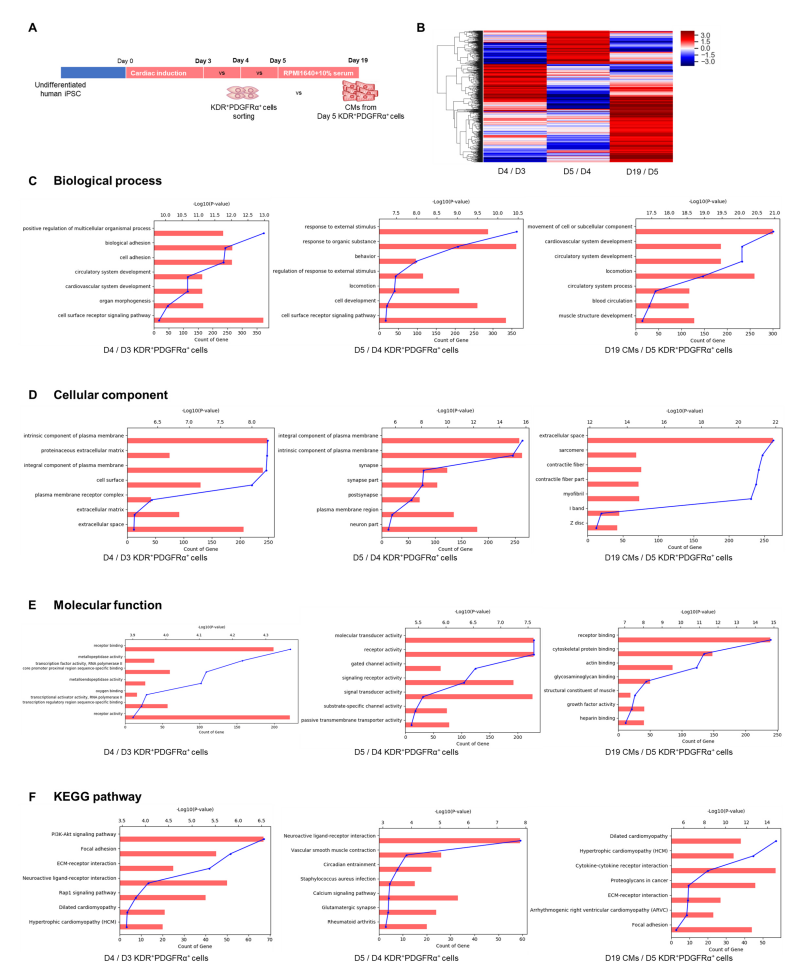

Fig. 2. Microarray transcriptome analysis of stage-specific cells during hiPSC-derived cardiac differentiation. (A) Sampling time points of hiPSC-drived cells: $\mathrm{KDR}^{+} \mathrm{PDGFR} \alpha^{+}$cells at days 3.0, 4.0, and 5.0 and cardiomyocytes at day 19.0. hiPSC: human induced pluripotent stem cell; KDR: kinase insert domain receptor; PDGFR $\alpha$ : platelet-derived growth factor receptor-alpha. (B) Heatmap of the hierarchical clustering comparison of each stage of cells derived from hiPSCs. (C-E) Gene Ontology analysis of the significantly upregulated genes in each developmental stage of cells derived from hiPSCs, showing the top 7 GO terms in the (C) Biological Process, (D) Cellular Component, and (E) Molecular Function categories. (F) Kyoto Encyclopedia of Genes and Genomes pathway analysis of the significantly upregulated genes in each stage of cells derived from hiPSCs; the top 7 pathways are shown. 
of Genes and Genomes (KEGG) pathway analyses were carried out to compare each stage of development in mESCs (Fig. 1C-F) and hiPSCs (Fig. 2C-F); the seven most high-ranking GO terms and KEGG pathways were selected. In the GO Biological Process category (Fig. $1 \mathrm{C}$ and $2 \mathrm{C}$ ), the significantly upregulated genes at cardiac lineage commitment were enriched in terms associated with responses to organic substances and external stimuli, as well as muscle and cardiovascular system development. Genes related to the heart contraction and circulatory system processes were significantly upregulated in the differentiated cardiomyocytes. In the Cellular Component category (Fig. 1D and 2D), the significantly upregulated genes were associated with extracellular region and plasma membrane components at cardiac lineage commitment. Genes related to myocardial contractile components, such as the sarcomere, Z disc, I band, and neuron parts, were found to be gradually upregulated during cardiomyocyte differentiation. In the Molecular Function category (Fig. $1 \mathrm{E}$ and $2 \mathrm{E}$ ), genes related to receptor binding and activity, gated channel activity, and cytoskeletal protein binding were significantly upregulated at cardiac lineage commitment and during cardiomyocyte differentiation. Pathway analysis (Fig. 1F and $2 \mathrm{~F}$ ) revealed that the significantly upregulated genes at cardiac lineage commitment and during cardiomyocyte differentiation were associated with phosphatidylinositol 3-kinase and protein kinase $\mathrm{B}$ (PI3K-AKT) signaling, extracellular matrix (ECM)-receptor interactions, calcium signaling, and cardiac hypertrophy and dilation pathways.

\section{Protein-protein interaction networks during cardiac differentiation}

We conducted protein-protein interaction analysis to gain insight into the regulation of cardiac lineage commitment, fate determination, and maturation during cardiac differentiation. In the mESC-derived cells, commonly expressed genes that showed over a 3-fold increase in expression levels in PDGFR $\alpha^{+}$CLCs were compared to those of vascular endothelial growth factor receptor 2-expressing $\left(\mathrm{Flk} 1^{+}\right)$mesodermal precursor cells (MPCs) and PDGFR $\alpha^{+}$cells without cardiac induction; these genes were selected first for analysis. Among the selected genes, genes that showed over 3-fold increased or decreased expression levels in the alpha-myosin heavy chain $(\alpha \mathrm{MHC})^{+}$cardiomyocytes were defined as the genes that induced cardiac maturation or the genes in control of cardiac linage commitment, respectively. When compared to the $\mathrm{Flk}^{+}$MPCs and PDGFR $\alpha^{+}$cells without cardiac induction, 425 genes showed over a 3-fold increase in expression levels in the PDGFR $\alpha^{+}$CLCs, of which 162 genes showed consistently increased expression levels in the $\alpha \mathrm{MHC}^{+}$cardiomyocytes and 89 genes showed decreased expression levels (Fig. 3A, Supplementary Table 1). The protein-protein interaction network was constructed using these 251 genes and the final mESC-derived cardiac differentiation network included 208 genes (Fig. 3B and C). When compared to the $\mathrm{Flk} 1^{+}$MPCs and

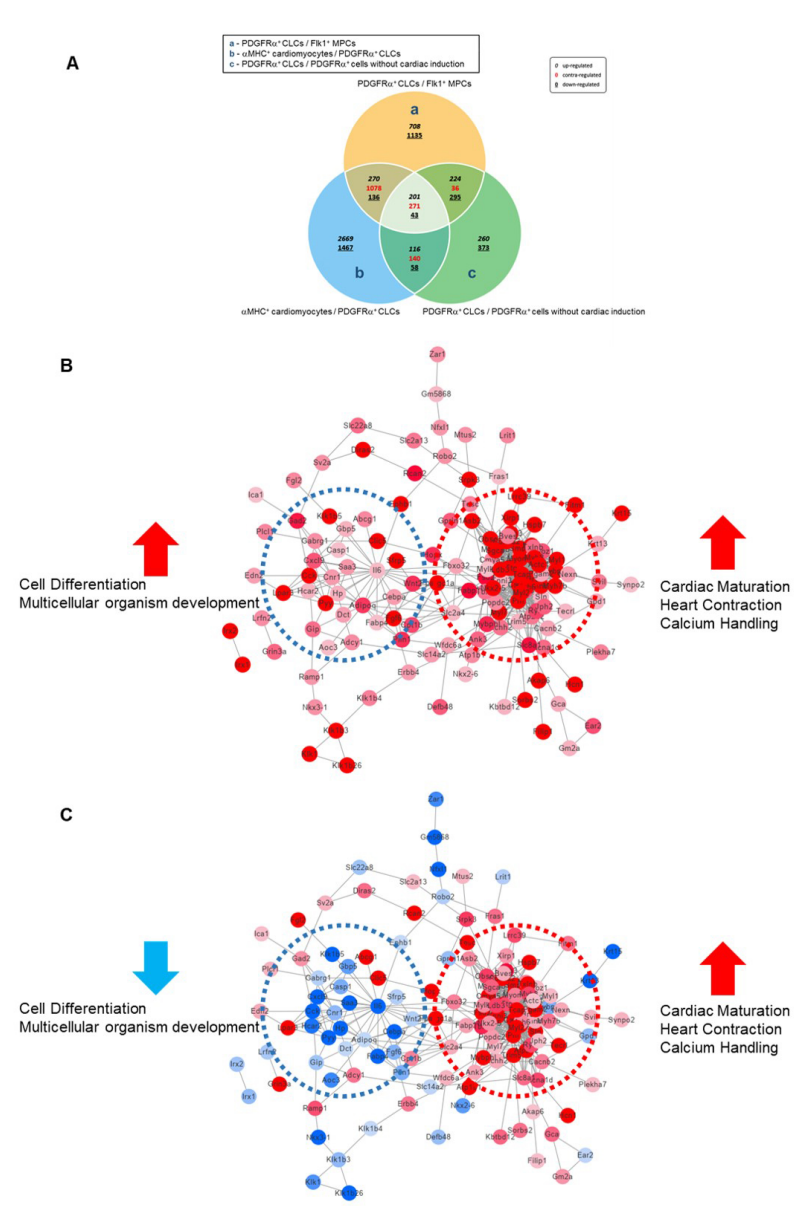

Fig. 3. Protein-protein interaction networks of mESC-derived cardiac differentiation, based on STRING analysis. (A) Venn diagrams depicting overlap of genes at each developmental stage of cells derived from mESCs during cardiac differentiation. mESC: murine embryonic stem cell; (B) Protein-protein interaction networks of commonly increased genes in the PDGFR $\alpha^{+} \mathrm{CLCs}$ as compared to $\mathrm{Flk}^{+}$MPCs and PDGFR $\alpha^{+}$cells without cardiac induction. Flk1 ${ }^{+}$MPCs: vascular endothelial growth factor receptor 2-expressing mesodermal precursor cells; PDGFR $\alpha^{+} \mathrm{CLCs}$ : platelet-derived growth factor receptor-alpha-expressing cardiac lineage-committed cells; $\alpha \mathrm{MHC}$ : alpha-myosin heavy chain. (C) Protein-protein interaction networks of consistently increased genes (red) and decreased genes (blue) in $\alpha \mathrm{MHC}^{+}$cardiomyocytes among the commonly increased genes in PDGFR $\alpha^{+}$CLCs.

$\mathrm{PDGFR}^{+}$cells without cardiac induction, the genes that commonly showed increased expression levels in the PDGFR $\alpha^{+}$ CLCs (Fig. 3B) were divided into genes with either increased (red) or decreased (blue) expression in the $\mathrm{\alpha MHC}^{+}$cardiomyocytes (Fig 3C). The genes with increased expression levels were mainly involved in regulating cardiac maturation, heart contraction, and calcium handling, and the genes with decreased expression levels were involved in regulating processes such as cell differentiation and multicellular 
organism development. Notably, these analyses revealed, for the first time, the cardiac developmental-immune-metabolic protein-protein interaction networks during cardiac lineage commitment. In the mESC-derived PDGFR $\alpha^{+} \mathrm{CLCs}$, the proteins Wnt family member 2 (Wnt2) and secreted frizzled related protein (Sfrp5) (related to cardiac development), interleukin 6 (II6) (related to immune system) and adiponectin (Adipoq), fatty acid-binding protein 4 (Fabp4) and hydroxycarboxylic acid receptor 2 (Hcar2) (related to metabolism) were connected.

In the hiPSC-derived cells, genes with consitently increased expression levels (over 2-fold) in the $\mathrm{KDR}^{+} \mathrm{PDGFR}^{+}$cells at day 4.0 as compared to those on day 3.0 and the genes with increased expression in the $\mathrm{KDR}^{+} \mathrm{PDGFR}^{+} \mathrm{CPC}$ compared to those of the $\mathrm{KDR}^{+} \mathrm{PDGFR} \alpha^{+}$cells at day 4.0 were selected for analyses depending on the time of differentiation. Among the selected genes, genes that showed over a 2-fold increase or decrease in expression levels in the cardiomyocytes were defined as the genes that induced cardiac maturation and the genes that controlled cardiac linage commitment, respectively. When compared to the $\mathrm{KDR}^{+} \mathrm{PDGFR} \alpha^{+}$cells at day 3.0 and 4.0, 296 genes showed over a 2-fold increase in expression level in the KDR ${ }^{+}$PDGFR $\alpha^{+}$CPCs, of which 104 genes showed continuous overexpression in the cardiomyocytes and 123 genes showed underexpression (Fig. 4A, Supplementary Table 2). The protein-protein interaction network was constructed using these 227 genes, and finally, an hiPSC-derived cardiac differentiation network composed of 191 genes was constructed (Fig. 4B and $\mathrm{C}$ ). When compared to the $\mathrm{KDR}^{+} \mathrm{PDGFR} \alpha^{+}$cells at day 3.0 and 4.0, the genes commonly overexpressed in the $\mathrm{KDR}^{+}$PDGFR $\alpha^{+}$CPCs (Fig. 4B) were divided into genes that were consistently overexpressed (red) or underexpressed (blue) in the cardiomyocytes (Fig 4C). Similar to the mESC-derived network, the genes showing increased expression levels were mainly involved in regulating cardiac maturation, heart contraction, and calcium handling, and the genes showing decreased expression levels were involved in regulating processes such as cell differentiation and multicellular organism development. In addition, the proteins fibroblast growth factor 10 (FGF10) (related to cardiac development), Toll-like receptor 4 (TLR4) (related to immune system), and alpha-2-HS-glycoprotein (AHSG) (related to metabolism) showed interactions in the hiPSC-derived $\mathrm{KDR}^{+} \mathrm{PDGFR}^{+} \mathrm{CPCs}$ and these data were similar to those of the mESC-derived network.

\section{DISCUSSION}

In this study, GO and KEGG pathway analyses revealed that genes related to the responses to organic substances and external stimuli, extracellular and myocardial contractile components, receptor binding, gated channel activity, PI3K-AKT signaling, and cardiac hypertrophy and dilation signaling pathways were commonly upregulated at cardiac lineage commitment. Protein-protein interaction network analysis revealed that genes that regulate cardiac maturation, heart

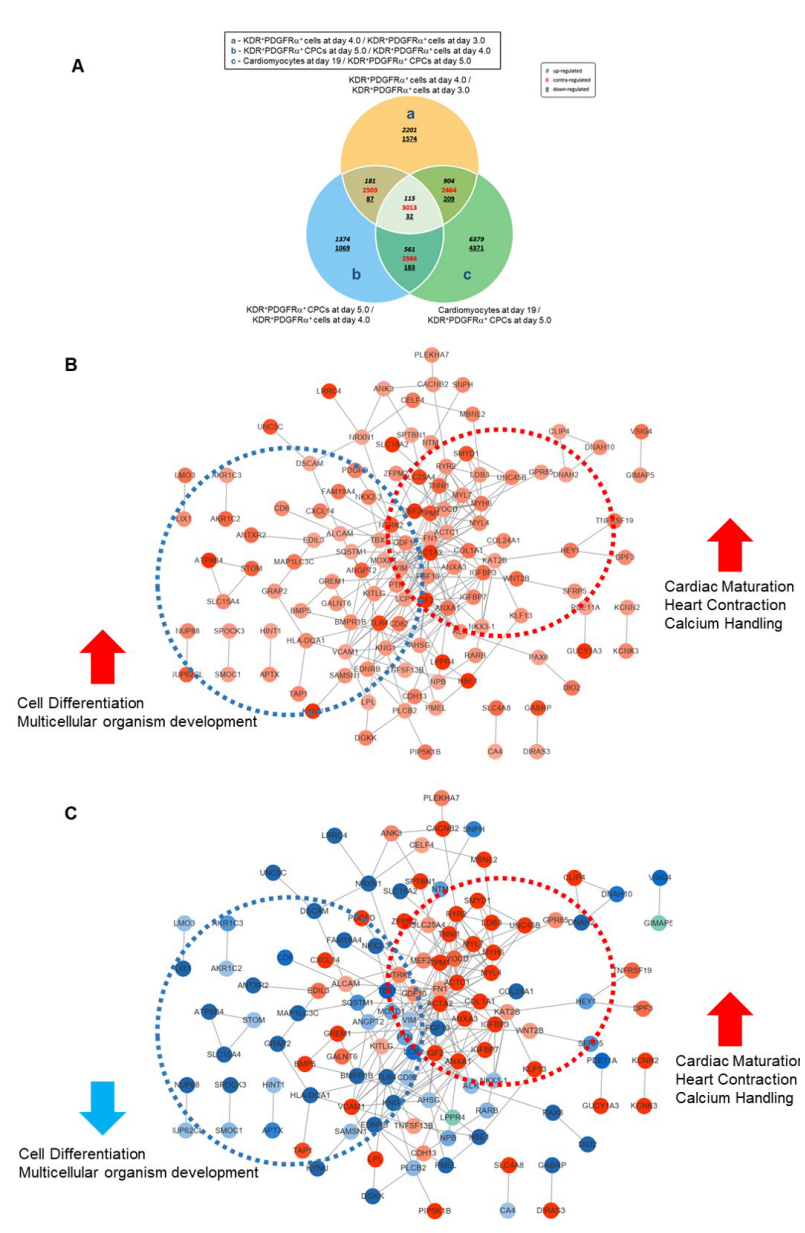

Fig. 4. Protein-protein interaction networks of hiPSC-derived cardiac differentiation, based on STRING analysis. (A) Venn diagrams depicting overlap of genes at each developmental stage of cells derived from hiPSCs during cardiac differentiation. hiPSC: human induced pluripotent stem cell. (B) Protein-protein interaction networks of commonly increased genes in $\mathrm{KDR}^{+} \mathrm{PDGFR} \alpha^{+} \mathrm{CPCs}$ at day 5.0 as compared to $\mathrm{KDR}^{+} \mathrm{PDGFR \alpha}{ }^{+}$cells at day 3.0 and 4.0. KDR: kinase insert domain receptor; PDGFR $\alpha$ : platelet-derived growth factor receptor-alpha. (C) Protein-protein interaction networks of consistently increased genes (red) and decreased genes (blue) in cardiomyocytes at day 19 as they relate to the commonly increased genes in $\mathrm{KDR}^{+} \mathrm{PDGFR} \alpha^{+} \mathrm{CPCs}$ at day 5.0.

contraction, and calcium handling were consistently increased during cardiac differentiation, while genes regulating cell differentiation and multicellular organism development were decreased at cardiac maturation after lineage commitment. Additionally, we identified for the first time the cardiac developmental-immune-metabolic protein-protein interaction networks in both the mESC-derived PDGFR $\alpha^{+} \mathrm{CLCs}$ (involving Wnt2, Sfrp5, Il6, Adipoq, Fabp4, and Hcar2) and hiPSCderived $\mathrm{KDR}^{+} \mathrm{PDGFR} \alpha^{+}$CPCs (involving FGF10, TLR4, and AHSG). 
To induce the differentiation of cardiomyocytes from PSCs, treatment with various compounds and growth factors at specific times and in specific doses is essential for directing mesodermal differentiation toward a more specific cardiac fate (1). Therefore, the cellular responses to organic substances and external stimuli, such as hormones, chemokines, cytokines, as well as cell adhesion-associated genes, may be significantly upregulated at cardiac lineage commitment. In order to receive signals from organic substances and external stimuli, the development of cell membrane structures and receptors is crucial. Indeed, our GO and KEGG pathway analyses revealed that genes associated with plasma membrane components and receptor binding and activity were significantly upregulated at cardiac lineage commitment.

Furthermore, it has been reported that cardiac ECM composition and structure, soluble factors, and extracellular vesicles may contribute to cardiomyocyte specification and maturation $(10,11)$. Interestingly, it was recently demonstrated that significant changes in the secretome profile occur during cardiac progenitor specification, and the complexity of the ECM increases (12). Consistent with these observation, our GO and KEGG pathway analyses revealed that genes associated with the extracellular region, ECM, and ECMreceptor interactions were highly upregulated at cardiac lineage commitment. Additionally, several studies have demonstrated that the PI3K-AKT signaling pathway contributes to cardiomyocyte differentiation, proliferation, and survival under developmental and pathological conditions (13-15). Similarly, our pathway analysis indicated several genes significantly upregulated at cardiac lineage commitment that were associated with PI3K-AKT signaling.

Our data also show that genes related to muscle and cardiovascular system development, blood circulation, myocardial contractile components (e.g., sarcomere, Z disc, and I band), cytoskeletal protein binding, and the cardiac hypertrophy and dilation pathways were consistently upregulated not only at cardiac lineage commitment, but also during further cardiomyocyte differentiation from both mESCs and hiPSCs. However, the genes associated with synapses, neuron parts, and calcium signaling pathways were expressed earlier in the hiPSC-derived $\mathrm{KDR}^{+} \mathrm{PDGFR} \alpha^{+} \mathrm{CPC}$; these genes were highly expressed in the mESC-derived differentiated $\alpha \mathrm{MHC}^{+}$ cardiomyocytes.

Notably, protein-protein interaction network analysis revealed two separate gene clusters, of which genes with consistently increased expression levels were involved in regulating cardiac maturation, heart contraction, and calcium handling during cardiac differentiation, whereas genes with decreased expression levels were involved in regulating cell differentiation and multicellular organism development at cardiac maturation following lineage commitment. These data suggest that the genes with decreased expression levels at cardiac maturation could contribute to cardiac lineage commitment and fate determination. Furthermore, we identified for the first time the cardiac developmental-immune-metabolic proteinprotein interaction networks in both the mESC-derived PDGFR $\alpha^{+}$ CLCs (involving Wnt2, Sfrp5, Il6, Adipoq, Fabp4, and Hcar2) and hiPSC-derived $\mathrm{KDR}^{+} \mathrm{PDGFR} \alpha^{+} \mathrm{CPCs}$ (involving FGF10, TLR4, and AHSG). These networks might have developed because PDGFR $\alpha^{+} \mathrm{CLCs}$ and $\mathrm{KDR}^{+} \mathrm{PDGFR} \alpha^{+} \mathrm{CPC}$ share some characteristics of other mesodermal lineage cells, such as immune cells and adipocytes. Although a few studies have reported that II6 contributes to cardiomyocyte-like cell differentiation from mesenchymal stem cells $(16,17)$, II6 and TLR4 are primarily inflammatory, immune-related proteins, and are mainly associated with cardiovascular diseases such as heart failure and atherosclerosis (18, 19). Similarly, Adipoq, Fabp4, Hcar2, and AHSG are associated with cardiometabolic diseases, including diabetes, obesity, heart failure, and coronary atherosclerosis (20-23). Therefore, we hypothesize that these cardiac developmental-immune-metabolic protein-protein interaction networks established at cardiac lineage commitment likely contribute to the future development of cardiometabolic diseases. Further studies using cardiometabolic disease- and patient-specific iPSC-derived cardiomyocytes are warranted to verify this hypothesis.

In the present study, we did not focus on the transcriptomic differences between mESC- and hiPSC-derived cardiomyocytes. Notably, a recent study showed that in terms of transcriptomes, mouse hearts at P0-3 and human hearts at 18-19 weeks post conception showed the closest proximity, and ribosomal genes showed differential expression patterns between mice and humans (24). Therefore, it may be worthwhile to analyze the transcriptomic differences between mESC- and hiPSC-derived cardiomyocytes across specific time points.

In conclusion, we analyzed the transcriptome-wide profiles of mESC-derived PDGFR $\alpha^{+} \mathrm{CLCs}$ and hiPSC-derived $\mathrm{KDR}^{+} \mathrm{PDGFR \alpha}^{+}$ CPCs with a focus on gene expression at cardiac lineage commitment. In this study, our group identified, for the first time, the cardiac developmental-immune-metabolic proteinprotein interaction networks of these cells.

\section{MATERIALS AND METHODS}

The detailed methods are described in the "Supplementary Materials and Methods".

\section{ACKNOWLEDGEMENTS}

This study was funded by the Basic Science Research Program through the National Research Foundation (NRF) of Korea funded by the Ministry of Education (2017R1D1A3B03034465), the NRF of Korea funded by the Ministry of Science and ICT (2020R1C1C1015104), and 2017 and 2018 Inje University research grants. 


\section{CONFLICTS OF INTEREST}

The authors have no conflicting interests.

\section{REFERENCES}

1. Burridge PW, Keller G, Gold JD, Wu JC (2012) Production of de novo cardiomyocytes: human pluripotent stem cell differentiation and direct reprogramming. Cell Stem Cell 10, 16-28

2. Cho SW, Park JS, Heo HJ et al (2014) Dual modulation of the mitochondrial permeability transition pore and redox signaling synergistically promotes cardiomyocyte differentiation from pluripotent stem cells. J Am Heart Assoc 3, e000693

3. Robertson C, Tran DD, George SC (2013) Concise review: maturation phases of human pluripotent stem cell-derived cardiomyocytes. Stem Cells 31, 829-837

4. Uosaki H, Cahan P, Lee DI et al (2015) Transcriptional landscape of cardiomyocyte maturation. Cell Rep 13, 1705-1716

5. Kattman SJ, Witty AD, Gagliardi M et al (2011) Stagespecific optimization of activin/nodal and BMP signaling promotes cardiac differentiation of mouse and human pluripotent stem cell lines. Cell Stem Cell 8, 228-240

6. Takeda M, Kanki Y, Masumoto $\mathrm{H}$ et al (2018) Identification of cardiomyocyte-fated progenitors from humaninduced pluripotent stem cells marked with CD82. Cell Rep 22, 546-556

7. Hong SP, Song S, Cho SW et al (2017) Generation of PDGFRalpha $(+)$ cardioblasts from pluripotent stem cells. Sci Rep 7, 41840

8. Hong SP, Song S, Lee S et al (2019) Regenerative potential of mouse embryonic stem cell-derived PDGFRalpha $(+)$ cardiac lineage committed cells in infarcted myocardium. World J Stem Cells 11, 44-54

9. Liu Q, Jiang C, Xu J et al (2017) Genome-wide temporal profiling of transcriptome and open chromatin of early cardiomyocyte differentiation derived from hiPSCs and hESCs. Circ Res 121, 376-391

10. Leitolis A, Robert AW, Pereira IT, Correa A, Stimamiglio MA (2019) Cardiomyogenesis modeling using pluripotent stem cells: the role of microenvironmental signaling. Front Cell Dev Biol 7, 164

11. Seo HR, Joo HJ, Kim DH et al (2017) Nanopillar surface topology promotes cardiomyocyte differentiation through cofilin-mediated cytoskeleton rearrangement. ACS Appl Mater Interfaces 9, 16803-16812
12. Robert AW, Pereira IT, Dallagiovanna B, Stimamiglio MA (2020) Secretome analysis performed during in vitro cardiac differentiation: discovering the cardiac microenvironment. Front Cell Dev Biol 8, 49

13. Naito AT, Akazawa $H$, Takano $H$ et al (2005) Phosphatidylinositol 3-kinase-Akt pathway plays a critical role in early cardiomyogenesis by regulating canonical Wnt signaling. Circ Res 97, 144-151

14. Samakova A, Gazova A, Sabova N, Valaskova S, Jurikova M, Kyselovic J (2019) The PI3k/Akt pathway is associated with angiogenesis, oxidative stress and survival of mesenchymal stem cells in pathophysiologic condition in ischemia. Physiol Res 68, S131-S138

15. Yang J, Savvatis K, Kang JS et al (2016) Targeting LOXL2 for cardiac interstitial fibrosis and heart failure treatment. Nat Commun 7, 13710

16. D'Amico MA, Ghinassi B, Izzicupo P, Di Ruscio A, Di Baldassarre A (2016) IL-6 activates PI3K and PKCzeta signaling and determines cardiac differentiation in rat embryonic H9c2 cells. J Cell Physiol 231, 576-586

17. Ibarra-lbarra BR, Franco M, Paez A, Lopez EV, Masso F (2019) Improved efficiency of cardiomyocyte-like cell differentiation from rat adipose tissue-derived mesenchymal stem cells with a directed differentiation protocol. Stem Cells Int 2019, 8940365

18. Bartekova $M$, Radosinska J, Jelemensky $M$, Dhalla NS (2018) Role of cytokines and inflammation in heart function during health and disease. Heart Fail Rev 23, 733-758

19. Yang $Y$, Lv J, Jiang $S$ et al (2016) The emerging role of toll-like receptor 4 in myocardial inflammation. Cell Death Dis 7, e2234

20. Zhang X, Cao YJ, Zhang HY, Cong H, Zhang J (2019) Associations between ADIPOQ polymorphisms and coronary artery disease: a meta-analysis. BMC Cardiovasc Disord 19,63

21. Furuhashi $M$ (2019) Fatty acid-binding protein 4 in cardiovascular and metabolic diseases. J Atheroscler Thromb 26, 216-232

22. Graff EC, Fang H, Wanders D, Judd RL (2016) Anti-inflammatory effects of the hydroxycarboxylic acid receptor 2. Metabolism 65, 102-113

23. Pan X, Wen SW, Bestman PL, Kaminga AC, Acheampong K, Liu A (2020) Fetuin-a in metabolic syndrome: a systematic review and meta-analysis. PLoS One 15, e0229776

24. Anzai T, Yamagata T, Uosaki H (2020) Comparative transcriptome landscape of mouse and human hearts. Front Cell Dev Biol 8, 268 